\title{
CAUCHY PROJECTORS ON NON-SMOOTH AND NON-RECTIFIABLE CURVES
}

\begin{abstract}
Let $f(t)$ be defined on a closed Jordan curve $\Gamma$ that divides the complex plane on two domains $D^{+}, D^{-}, \infty \in D^{-}$. Assume that it is representable as a difference $f(t)=F^{+}(t)-F^{-}(t)$, $t \in \Gamma$, where $F^{ \pm}(t)$ are limits of a holomorphic in $\overline{\mathbb{C}} \backslash \Gamma$ function $F(z)$ for $D^{ \pm} \ni z \rightarrow t \in \Gamma, F(\infty)=0$. The mappings $f \mapsto F^{ \pm}$are called Cauchy projectors.

Let $H_{\nu}(\Gamma)$ be the space of functions satisfying on $\Gamma$ the Hölder condition with exponent $\nu \in(0,1]$. It is well known that on any smooth (or piecewise-smooth) curve $\Gamma$ the Cauchy projectors map $H_{\nu}(\Gamma)$ onto itself for any $\nu \in(0,1)$, but for essentially non-smooth curves this proposition is not valid.

We will show that even for non-rectifiable curves the Cauchy projectors continuously map the intersection of all spaces $H_{\nu}(\Gamma)$, $0<\nu<1$ (considered as countably-normed Frechet space) onto itself.
\end{abstract}

Key words: Cauchy projectors, non-smooth curves, non-rectifiable curves

\section{Mathematical Subject Classification: 30E20}

1. Introduction. Let $\Gamma$ be a simple closed curve on the complex plane dividing it to two domains $D^{+}$and $D^{-}, \infty \in D^{-}$. If $\Gamma$ is rectifiable and a function $f(t)$ is integrable over it, then the Cauchy type integral

$$
\mathcal{C} f(z):=\frac{1}{2 \pi i} \int_{\Gamma} \frac{f(t) d t}{t-z}, \quad z \notin \Gamma .
$$

is defined. It represents an analytic in $\overline{\mathbb{C}} \backslash \Gamma$ function that vanishes at the infinity. Let this function have limit values on $\Gamma$ from both sides:

$$
\mathcal{C}^{ \pm} f(t):=\lim _{D^{ \pm} \ni z \rightarrow t \in \Gamma} \mathcal{C} f(z)
$$

(C) Petrozavodsk State University, 2019 
These limits are called the Cauchy projectors.

The Cauchy type integral and the Cauchy projectors are of importance for the whole complex analysis. Their applications in Riemann-Hilbert boundary value problems are described in well-known monographs [1-3]. A contemporary results on this subject can be found in the survey [4].

We consider the Hölder condition, i. e., the following restriction on a function $f$ :

$$
h_{\nu}(f ; \Gamma):=\sup \left\{\frac{\left|f\left(t_{1}\right)-f\left(t_{2}\right)\right|}{\left|t_{1}-t_{2}\right|^{\nu}}: t_{1,2} \in \Gamma, t_{1} \neq t_{2}\right\}<\infty,
$$

where the exponent $\nu$ is positive, $\nu \in(0,1]$. We denote by $H_{\nu}(\Gamma)$ the set of all functions that satisfy this condition. It is a Banach space with the norm

$$
\|f\|_{\nu}:=\sup \{|f(t)|: t \in \Gamma\}+h_{\nu}(f ; \Gamma) .
$$

If $\Gamma$ is a smooth curve and $f \in H_{\nu}(\Gamma)$, then the Cauchy projectors are representable as $\mathcal{C}^{ \pm} f=\frac{1}{2}(f \pm \mathcal{S} f)$, where

$$
\mathcal{S} f(t):=\frac{1}{\pi i} v \cdot p \cdot \int_{\Gamma} \frac{f(\tau) d \tau}{\tau-t}
$$

is a singular integral with the Cauchy kernel.

The following result belongs to the base of the theory of singular integral equations $[1,2]$. Recall that a Ljapunov curve is a curve with Höldercontinuous angle between its tangent and a fixed axis.

Theorem 1. ([5], p.199, item 4.5) Let a curve $\Gamma$ be smooth. If $f \in H_{\nu}(\Gamma)$, then the singular integral operator $\mathcal{S}$ maps $H_{\nu}(\Gamma), 0<\nu<1$, onto itself. If $\Gamma$ is a Ljapunov curve, then this mapping is bounded. If $f \in H_{1}(\Gamma)$, then $\mathcal{S}^{ \pm} f \in H_{\nu}(\Gamma)$ for any $\nu<1$.

Thus, the image of $H_{1}(\Gamma)$ is the set $H_{1-0}(\Gamma):=\bigcap_{\nu<1} H_{\nu}(\Gamma)$. It is not a Banach space, but any increasing sequence of exponents $\nu_{1}<\nu_{2}<\cdots<$ $<\nu_{k}<\ldots$ such that $\lim _{k \rightarrow \infty} \nu_{k}=1$ generates a family of semi-norms $h_{\nu_{k}}(\cdot ; \Gamma)$ in $H_{1-0}(\Gamma)$.

In 1979, E. M. Dyn'kin [6] and T. Salimov [7] independently proved the following result for rectifiable (but not necessarily smooth) curves.

Theorem 2. Let a curve $\Gamma$ be rectifiable. If $f \in H_{\nu}(\Gamma), \nu \in\left(\frac{1}{2}, 1\right]$, then the boundary values $\mathcal{C}^{ \pm} f$ exist and belong to $H_{\mu}(\Gamma)$ for any $\mu<2 \nu-1$, and this bound cannot be improved. 
We see that on rectifiable curves the projectors $\mathcal{C}^{ \pm}$do not map $H_{\nu}(\Gamma)$ onto itself for $0<\nu<1$, but they map $H_{1-0}(\Gamma)$ onto $H_{1-0}(\Gamma)$.

In this paper, we study the action of the Cauchy projectors on countably-normed space $H_{1-0}(\Gamma)$. In the sequel, we assume, without loss of generality, that the diameter of $\Gamma$ is less than unit.

2. The Hölder-Frechet space $\mathbf{H}_{\mathbf{1 - 0}}$. Let $A$ be a compact subset of the complex plane $\mathbb{C}$ with the diameter $d<1$. We denote bt $H_{1-0}(A)$ the set of all functions on $A$ that satisfy there the Hölder condition with any exponent less than 1 .

We consider a sequence $\tilde{\nu}=\left\{\nu_{k}\right\}_{k=0}^{\infty}$ of exponents such that $0<\nu_{0}<$ $<\nu_{1}<\cdots<\nu_{k}<\cdots<1, \lim _{k \rightarrow \infty} \nu_{k}=1$, and norms

$$
\|f\|_{k}:=\sup \{|f(t)|: t \in A\}+h_{\nu_{k}}(f ; A), \quad k=0,1, \ldots,
$$

and denote by $H_{1-0}(A ; \tilde{\nu})$ the set $H_{1-0}(A)$ equipped with these norms. As $d<1$,

$$
\frac{\left|f\left(t_{1}\right)-f\left(t_{2}\right)\right|}{\left|t_{1}-t_{2}\right|^{\nu_{k+1}}}>\frac{\left|f\left(t_{1}\right)-f\left(t_{2}\right)\right|}{\left|t_{1}-t_{2}\right|^{\nu_{k}}}, \quad t_{1}, t_{2} \in A,
$$

i. e., the sequence of norms increases. Obviously, any two norms (4) are coordinated (see [8]), and $H_{1-0}(A ; \tilde{\nu})$ is a countably-normed Frechet space.

If $\Gamma$ is a Ljapunov curve, then all mappings $\mathcal{C}^{ \pm}, \mathcal{S}$ are bounded linear operators $H_{1-0}(\Gamma ; \tilde{\nu}) \mapsto H_{1-0}(\Gamma ; \tilde{\nu})$. This fact follows from the proof of Theorem 1 .

There are many examples of compact integral operators in the space $H_{1-0}(\Gamma)$. For instance, the operator

$$
K f:=\int_{\Gamma} f(\tau) k(\tau, t) d \tau,
$$

is compact (see $[9,10])$, if its kernel $k(\tau, t)$ is continuous and satisfies the following condition of Hölder type:

$$
\left|k\left(\tau, t_{1}\right)-k\left(\tau, t_{2}\right)\right| \leqslant c(\tau)\left|t_{1}-t_{2}\right|, \quad \tau, t_{1}, t_{2} \in \Gamma,
$$

and the function $c(\tau)$ is integrable over $\Gamma$. Then the singular integral equation

$$
a(t) f(t)+b(t) \mathcal{S} f(t)+K f(t)=c(t), \quad t \in \Gamma,
$$

with the desired function $f$ and coefficients $a, b, c \in H_{1-0}(\Gamma ; \tilde{\nu})$ keeps in the space $H_{1-0}(\Gamma ; \tilde{\nu})$ all its classic properties $[1,2]$. 
3. Non-smooth rectifiable curves. We consider non-smooth rectifiable curves $\Gamma$.

Theorem 3. If a simple closed curve $\Gamma$ is rectifiable and a sequence $\tilde{\nu}$ satisfies inequalities

$$
\nu_{0}>\frac{1}{2}, \quad \nu_{k+1}>\frac{1+\nu_{k}}{2}, \quad k=0,1, \ldots,
$$

then the mappings $\mathcal{C}^{ \pm}$are bounded linear operators

$$
\mathcal{C}^{ \pm}: H_{1-0}(\Gamma ; \tilde{\nu}) \rightarrow H_{1-0}(\Gamma ; \tilde{\nu}) .
$$

Proof. Let $f \in H_{1-0}(\Gamma ; \tilde{\nu})$. We apply the Whitney extension operator $\mathcal{E}_{0}$. As it is well-known (see, for instance, [11]), the obtained extension has the following properties:

- the function $\mathcal{E}_{0} f$ is defined in the whole complex plane and $\left.\mathcal{E}_{0} f\right|_{\Gamma}=f$;

- if $f \in H_{\nu}(\Gamma)$, then $\mathcal{E}_{0} f \in H_{\nu}(\mathbb{C})$, and $h_{\nu}\left(\mathcal{E}_{0} f ; \mathbb{C}\right)=h_{\nu}(f ; \Gamma)$;

- the function $\mathcal{E}_{0} f$ is differentiable with respect to real variables $x$ and $y$ in $\mathbb{C} \backslash \Gamma$, and its first partial derivatives satisfy the estimate

$$
\left|\nabla \mathcal{E}_{0} f(z)\right| \leqslant \frac{h_{\nu}(f ; \Gamma)}{\operatorname{dist}^{1-\nu}(z, \Gamma)}, \quad z \notin \Gamma .
$$

In the considered situation the last two properties are valid for any $\nu<1$. We consider function $u(z)$ that equals $\mathcal{E}_{0} f(z)$ for $z \in D^{+}$and to 0 for $z \in D^{-}$. The Cauchy type integral can be represented by means of the Stokes formula, as follows:

$$
\mathcal{C} f(z)=u(z)-\frac{1}{2 \pi i} \iint_{D^{+}} \frac{\partial u}{\partial \bar{\zeta}} \frac{d \zeta d \bar{\zeta}}{\zeta-z}, \quad z \in \mathbb{C} \backslash \Gamma .
$$

As the curve $\Gamma$ is rectifiable, the function $\operatorname{dist}^{-p}(z ; \Gamma)$ is integrable in $D^{+}$ for any $p<1$ (see, for instance, [12]). Therefore, the derivative $\frac{\partial u}{\partial \bar{z}}$ is integrable in $D^{+}$with an arbitrarily large power $p$, and $\|\partial u / \partial \bar{z}\|_{L^{p}\left(D^{+}\right)} \leqslant C h_{\nu}(f ; \Gamma)$ for any $\nu<1$ and $p(1-\nu)<1$. Here and in what follows the letter $C$ stands for a positive value, which depends on $\nu, p$ and $\Gamma$, but does not depend on $f$. Then we apply the well-known property of the integral operator

$$
\phi \rightarrow T \phi:=\iint_{D^{+}} \frac{\phi(\zeta) d \zeta d \bar{\zeta}}{\zeta-z}
$$


(see, for instance, [13]): if $\phi \in L^{p}\left(D^{+}\right), p>2$, then $T \phi \in H_{1-\frac{2}{p}}(\mathbb{C})$ and $h_{1-\frac{2}{p}}(T \phi ; \mathbb{C}) \leqslant C\|\partial u / \partial \bar{z}\|_{L^{p}\left(D^{+}\right)}$. Thus, $\left\|\mathcal{C}^{ \pm} f\right\|_{k+1} \leqslant C\|f\|_{k}$.

The singular integral looses some of its properties at the points where $\Gamma$ is not smooth. In this connection, we consider the equation

$$
a(t) \mathcal{C}^{+} f(t)+b(t) \mathcal{C}^{-} f(t)+K f(t)=c(t), \quad t \in \Gamma,
$$

with $a, b, c \in H_{1-0}(\Gamma ; \tilde{\nu})$ and the same operator $K$. Clearly, it also keeps the classic properties of singular integral equations in the space $H_{1-0}(\Gamma ; \tilde{\nu})$.

4. Non-rectifiable curves. Let a simple closed curve $\Gamma$ be not rectifiable. Then, the curvilinear integrals over that curve are undefined, and the formulas (1) and (2) loose their sense. However, we are able to define the Cauchy projectors in the following way.

We denote by $\operatorname{dmh} A$ and $\overline{\operatorname{dm}} A$ the Hausdorff dimension and the upper Minkowskii dimension of a compact set $A \subset \mathbb{C}$, correspondingly (see, for instance, [14]). Consider the jump problem, i. e., the problem of evaluation of a holomorphic in $\overline{\mathbb{C}} \backslash \Gamma$ function $\Phi(z)$ that satisfies the conditions

(J1) $\Phi$ has the limit values $\Phi^{ \pm}(t)$ from both sides at any point $t \in \Gamma$, and these values are connected by the equality

$$
\Phi^{+}(t)-\Phi^{-}(t)=f(t), \quad t \in \Gamma
$$

(J2) $\Phi(\infty)=0$.

If $\mathrm{dmh} \Gamma>1$, then a solution of this problem cannot be unique, because in this case there exists a non-trivial function that is continuous in $\overline{\mathbb{C}}$ and holomorphic in $\overline{\mathbb{C}} \backslash \Gamma$ (see [15]). However, the E. P. Dolzhenko theorem [15] states: if a function $\Phi$ satisfies the Hölder condition with the exponent $\mu>\operatorname{dmh} \Gamma-1$ in a domain $D \supset \Gamma$ and is holomorphic in $D \backslash \Gamma$, then it is holomorphic in $D$.

Therefore, we supply the formulation of the jump problem by the condition

(J3) $\Phi^{ \pm} \in H_{\mu}(\Gamma), \mu>\operatorname{dmh} \Gamma-1$,

and the problem $(\mathrm{J} 1),(\mathrm{J} 2),(\mathrm{J} 3)$ cannot have more than one solution. If its unique solution exists, then we put

$$
\mathcal{C}^{ \pm} f:=\Phi^{ \pm}
$$

we keep the notation $\mathcal{C}^{ \pm}$, because on smooth curves both definitions of the Cauchy projectors coincide. 
Theorem 4. If a simple closed non-rectifiable curve $\Gamma$ satisfies the condition $\overline{\operatorname{dm}} \Gamma<2$, and a sequence $\tilde{\nu}$ satisfies inequalities

$$
\nu_{0}>\frac{1}{2} \overline{\operatorname{dm}} \Gamma, \quad \nu_{k+1}>\frac{\overline{\mathrm{dm}} \Gamma+(2-\overline{\mathrm{dm}} \Gamma) \nu_{k}}{2}, \quad k=0,1, \ldots
$$

then the boundary values $\mathcal{C}^{ \pm}$exist and are bounded linear operators from $H_{1-0}(\Gamma ; \tilde{\nu})$ into itself.

Proof. The solvability of the jump problem (J1), (J2), (J3) is studied in [12]. There it is proved that the function $\operatorname{dist}^{-p}(z ; \Gamma)$ is integrable in $D^{+}$for any $p<2-\overline{\mathrm{dm}} \Gamma$. The substitution of this estimate into the consideration of the previous section concludes the proof.

Acknowledgment. The research of the first author is partially supported by the Russian Foundation for Basic Researches and the Government of Republic Tatarstan, grant 18-41-160003 r-a.

\section{References}

[1] Gakhov F. D. Boundary value problems. Moscow, Nauka, 1977.

[2] Muskhelishvili N. I. Singular integral equations. Moscow, Nauka, 1962.

[3] Jian-Ke Lu. Boundary value problems for analytic functions. Singapore, World Scientific, 1993.

[4] Abreu-Blaya R., Bory-Reyes J., Kats B. A. The Cauchy Type Integral and Singular Integral Operator over closed Jordan curves. Monatshefte für Mathematik, 2015, vol. 176, issue 1, pp. 1-15. DOI: https://doi.org/ 10.1007/s00605-014-0656-9.

[5] Privalov I. I. Boundary properties of analytic functions. Moscow, GITTL, 1950.

[6] Dyn'kin E. M. Smoothness of the Cauchy type integral. Zapiski nauchnyh semin. LOMI AN USSR, 1979, 92, pp. 115-133.

[7] Salimov T. Direct estimate for singular Cauchy integral over closed path. Nauchn. trudy MV i SSO Azerb. SSR, 1979, no. 5, pp. 59-75.

[8] Gel'fand I. M., Shilov G. E. Spaces of test and generalized functions. Moscow, Physmathgiz, 1958.

[9] Gorin S. V. Fredholm property of singular operators in spaces of infinitely differentiable vector-functions. cand. diss., Rostov-na-Donu, 2017.

[10] Gorin S. V. An algebra of singular operators in space of smooth functions. Izvestija vuzov. Sev.-Caucas Region. Ser. Estestv. Nauki. 2006, no. 1., pp. $21-28$. 
[11] Stein E. M. Singular integrals and differential properties of functions. Princeton, Princeton University Press, 1970.

[12] Kats B. A. Riemann problem on a closed Jordan curve. Izvestija vuzov. Matem. 1983, no. 4, pp. 68-80.

[13] Vekua, I. N. Generalized analytical functions. Moscow, Nauka, 1988.

[14] Falconer K. J. Fractal geometry. Wiley and Sons, 3rd edition, 2014.

[15] Dolzhenko E. P. On "erazing" of singularities of analytic functions. Uspekhi Matem. Nauk, 18(1963), no. 4, pp. 135-142.

Received July 28, 2018.

In revised form, December 21, 2018.

Accepted December 24, 2018.

Published online January 5, 2019.

Kazan Federal University

18 Kremlyovskaya str., Kazan 420008, Russia

E-mail: katsboris877@gmail.com 